\section{Comportamento sexual e contracepção de emergência entre adolescentes de escolas públicas de Pernambuco, Brasil}

\author{
Sexual behavior and emergency contraception \\ among adolescents from public schools in \\ Pernambuco State, Brazil
}

\section{${ }^{1}$ Faculdade de Enfermagem Nossa Senhora das Graças, Universidade de Pernambuco, Recife, Brasil. \\ 2 Faculdade de Ciências Médicas, Universidade de Pernambuco, Recife, Brasil. \\ Correspondência M. S. P. Araújo Faculdade de Enfermagem Nossa Senhora das Graças, Universidade de Pernambuco. Rua Uriel Paes Barreto 53 apto. 702, Recife, $P E$ 50710-500, Brasil. suelypeixoto2004@yahoo. com.br}

\begin{abstract}
This study focused on knowledge and use of emergency contraception among 4,210 adolescents (14-19 years) enrolled in public schools in Pernambuco State, Brazil. Information was collected using the Global School-Based Student Health Survey, previously validated. Knowledge, frequency, and form of use of emergency contraception were investigated. Independent variables were classified as socio-demographic and those related to sexual behavior. Most adolescents reported knowing and having received information about the method, but among those who had already used it, only $22.1 \%$ had done so correctly. Adjusted regression analysis showed greater likelihood of knowledge about the method among girls (OR = 5.03; 95\%CI: 1.72-14.69) and the sexually initiated (OR = 1.52; 95\% CI: 1.34-1.75), while rural residents were $68 \%$ less knowledgeable. Rural residents showed 1.68 times higher odds (CI95\%: 1.09-2.25) of incorrect use, while girls showed $71 \%$ lower likelihood of incorrect use. Sexual and reproductive education is necessary, especially among male and rural adolescents.
\end{abstract}

Contraception; Adolescent; Sexual Behavior
Maria Suely Peixoto de Araújo 1

Laura Olinda Bregieiro Fernandes Costa 2

\section{Introdução}

A adolescência tem seu conceito determinado pela história de cada sociedade que imprime marcas nesse período cronológico, influenciadas pelos diferentes processos biopsíquicos e sócioculturais peculiares aos grupos nos quais os indivíduos estão inseridos. Por isso, a Organização Mundial da Saúde (OMS) define adolescência de forma abrangente, como o período entre os $10 \mathrm{e}$ 19 anos de idade 1 .

Uma das características da adolescência é o início de vida sexual como forma de participação das relações de poder entre os gêneros, assim como a formação da identidade social. Tal exercício da sexualidade tem sido feito à custa de um grande número de gravidezes não planejadas, em todo o mundo, quer por erro, por não-utilização ou falha dos métodos contraceptivos, ou mesmo por violência sexual 2 .

Considerando esses riscos, a contracepção de emergência se apresenta como uma alternativa válida, porém condicionada ao disciplinamento. As formulações hormonais de progesterona isolada ou combinada a estrógenos (método Yuspe), habitualmente usadas para contracepção de emergência, são efetivas quando tomadas decorridas $72 \mathrm{~h}$ a $120 \mathrm{~h}$ pós-coito, no entanto têm maior efetividade quando a primeira dose é administrada nas primeiras $12 \mathrm{~h}$, ou, na opção de dose única, seguindo o mesmo critério. Uma gestação inoportuna oferece risco, 
cada vez maior, quanto mais distante estiver a ingestão da medicação do ato sexual desprotegido de métodos anticoncepcionais ${ }^{3}$.

Dentre as características técnicas do uso de contracepção de emergência, estão as recomendações para cautela na administração de hormônios, especialmente quando os indivíduos têm predisposição genética para trombose, sugerindo estudos mais profundos sobre o mecanismo de ação. Além disso, alguns questionamentos têm sido levantados em relação aos efeitos colaterais dos hormônios (método Yuspe e levonorgestrel), embora mais pronunciada com as combinações hormonais contendo estrógeno, incluindo náuseas, vômitos, sensibilidade mamária, sangramentos irregulares e cefaléia, assim como alterações na hemostasia.

Ao analisar as causas da exposição ao risco de gestação indesejada, as hipóteses perpassam a ausência de um programa de educação sexual 4,5, vergonha de ir ao médico, preconceito quanto à utilização de preservativos, falta de informação e dificuldade de obtenção dos métodos contraceptivos de barreira e hormonal 6,7,8 e, por vezes, pela aceitação do jogo de poder de gênero, com submissão da mulher aos padrões sociais de domínio 9,10.

A interação entre o biológico, o social e o simbólico gera conflito na busca de autonomia nas questões da saúde reprodutiva, pois o adolescente trata a gravidez indesejada e a doença sexualmente transmissível como algo improvável de acontecer. Outro fator que contribui para que esses desfechos possam ocorrer está relacionado ao imaginário social de que a maternidade é um imperativo do sexo feminino 11,12.

A contracepção de emergência também vem atender à falha desse imaginário, já que as adolescentes que intencionalmente engravidam, porque planejam, representam uma pequena parcela desse grupo populacional. Mesmo assim, esse desejo tem sido atribuído por alguns autores a uma forte influência do social 13,14.

Essas suposições ganham especial importância quando se constata que baixas condições sócio-econômicas e de escolaridade, em países em desenvolvimento como o Brasil, podem atuar como fatores de risco para gravidez indesejada 15. Entretanto também podem estar mais relacionadas às práticas sexuais do que ao conhecimento formal sobre métodos contraceptivos 16,17 .

Embora a contracepção de emergência seja reconhecida em muitos países como direito relacionado à saúde reprodutiva, sua subutilização pode depender do desconhecimento e do conflito ético-religioso. Por conta disso, talvez algumas mulheres só consigam as formulações e orien- tações nas emergências, em caso de violência sexual 18 .

A presente pesquisa tem por objetivo descrever o comportamento sexual dos adolescentes de escolas públicas estaduais de Pernambuco, seu conhecimento sobre a contracepção de emergência e a forma de sua utilização.

\section{Sujeitos e métodos}

O presente estudo faz parte do projeto Estilos de Vida e Comportamento de Risco à Saúde do Estudante do Ensino Médio de Pernambuco: do Diagnóstico à Intervenção, desenvolvido pelo Grupo de Pesquisa em Estilos de Vida e Saúde, Escola Superior de Educação Física, Universidade de Pernambuco, em cooperação com a Secretaria de Educação e Cultura do Estado de Pernambuco (SEDUC), para que a população alvo fosse limitada aos estudantes da rede pública estadual de Ensino Médio, os quais em 2005 representavam 352.829 sujeitos 19.

O cálculo amostral e o dimensionamento da amostra foram efetuados de forma a atender aos diversos objetivos do projeto, que incluíam avaliação de dez módulos de comportamentos de risco relacionados à saúde (consumo de álcool e outras drogas, atividade física, hábitos alimentares, comportamento sexual, violência, higiene, tabagismo, saúde mental e relacionamentos). Por esse motivo, para o cálculo amostral foram utilizados os critérios: população estimada em 352.829 sujeitos; intervalo de $95 \%$ de confiança; erro de 3 pontos percentuais; prevalência estimada em 50\% (para os diversos comportamentos) e efeito de delineamento estabelecido em quatro vezes o tamanho mínimo da amostra. A literatura especializada sugere que, em virtude da opção por método de amostragem por conglomerados, um efeito de delineamento de 1,4 a 1,5 seria suficiente para resguardar a precisão desejada 20 . Contudo, pesquisadores têm empregado efeito de delineamento superior a este valor em estudos nacionais 21 , do que derivou a decisão de se aplicar uma correção mais conservadora ao tamanho mínimo da amostra. Adicionalmente, visando atenuar as limitações impostas por eventuais perdas na aplicação por recusa, preenchimento inadequado dos questionários, baixa freqüência às aulas e alunos fora de faixa escolar, decidiu-se por aumentar em $45 \%$ o tamanho da amostra. Assim, o plano amostral foi elaborado com a intenção de se alcançar uma amostra de aproximadamente 6.114 estudantes, estimandose 4.264 estudantes com idade de 14 a 19 anos, considerada critério de inclusão associado à autorização dos pais. 
Para o presente estudo, foram visitadas 76 escolas (11\% do total de escolas estaduais) em 44 municípios, o que representou $23 \%$ do total de municípios pernambucanos. Foram entrevistados 4.210 estudantes do total de 4.264 previstos (1,3\% de perdas e recusas).

Procurou-se garantir que a amostra estudada tivesse uma proporção semelhante de estudantes distribuídos conforme região geográfica, tamanho da escola e turno das aulas. A distribuição regional foi observada pelo número de estudantes matriculados em cada uma das 17 Gerências Regionais de Ensino. O tamanho da escola foi classificado em três níveis de acordo com o número de alunos matriculados no ensino médio, observando-se os critérios: porte I (pequeno) - menos de 200 alunos; porte II (médio) - 200 a 499 alunos e porte III (grande) - 500 alunos ou mais. Alunos matriculados no turno da manhã e da tarde foram agrupados numa única categoria (estudantes do período diurno).

Tendo em conta tais critérios, recorreu-se a um procedimento de amostragem por conglomerados em dois estágios para seleção da amostra requerida. No primeiro estágio, todas as escolas estaduais de Pernambuco foram consideradas elegíveis $(n=668)$. A seleção foi aleatória e estratificada, adotando-se como critérios de estratificação: densidade da escola e estudantes de cada microrregião do Estado e porte da escola (pequeno médio e grande). Ao final, 76 escolas foram sorteadas para o estudo. No segundo estágio, todas as turmas das escolas sorteadas foram elegíveis $(n=8.019)$. A seleção foi aleatória simples e como critério de estratificação, considerou-se a densidade de turmas e turno diurno ou noturno. Ao final, 203 turmas foram sorteadas para o estudo. Todos os sorteios foram realizados por meio de números aleatórios no programa Randomizer (Social Psychology Network, Middletow, Estados Unidos; http://randomizer.org/index.htm).

Antecedendo a coleta de dados, o Termo de Consentimento Livre e Esclarecido Negativo foi encaminhado via malote para cada uma das escolas sorteadas para que a Direção enviasse a todos os pais, a fim de que o assinassem e remetessem de volta à escola, no caso de não autorizar a participação de seu filho.

O questionário utilizado foi uma versão traduzida e previamente testada do Global School-Based Student Health Survey (GSHS), proposto pela OMS em colaboração com o Fundo das Nações Unidas para a Infância e o Programa Conjunto das Nações Unidas sobre HIV/AIDS, juntamente com a assistência técnica do Center for Disease Control and Prevention, disponível no endereço eletrônico (http://www.who.int/chp/gshs/en).
Na versão em português, além das questões originais do questionário, foram incluídas na sessão de comportamento sexual perguntas relacionadas ao contraceptivo de emergência. Para teste e validação do instrumento, foi realizado um estudo piloto com 155 estudantes de nível médio de uma escola pública do Município de Recife. Indicadores de reprodutibilidade (consistência de medidas teste-reteste) foram de moderados a altos na maioria dos itens do instrumento, e os coeficientes de concordância (índice kappa) para as variáveis de comportamento sexual e contracepção de emergência variaram (os valores) de 0,83 a 1,00 .

A coleta dos dados foi realizada no período de abril a outubro de 2006. A aplicação do questionário foi efetuada por seis mestrandos do programa de pós-graduação em Hebiatria da Universidade de Pernambuco, que participaram de uma capacitação prévia para padronização dos procedimentos de coleta de dados. O questionário foi auto-responsivo, após leitura em voz alta de cada questão feita pelo aplicador.

As variáveis foram agrupadas em: sóciodemográficas (sexo, estado civil, cor da pele, ocupação do adolescente, local de residência, escolaridade materna e posse de computador); de comportamento sexual (referência ao início de vida sexual, idade da primeira relação sexual expressa em anos, uso de preservativo na última relação sexual, número de parceiros sexuais durante a vida) e de contracepção de emergência (agentes de informação, freqüência de uso do método nos últimos em 12 meses e forma de uso). Admitiu-se como correto o uso de contracepção de emergência iniciado nas primeiras 72 horas pós-coito, independente de método, esquema e formulação empregada.

Os dados foram tabulados no programa Epidata, versão 3.1 (Epidata Association, Odense, Dinamarca), com identificação de erros de digitação por meio de dupla entrada de dados. Para a obtenção dos cálculos estatísticos, foram utilizados os programas SAS (SAS Inst., Cary, Estados Unidos), versão 8, e SPSS versão 13.0 (SPSS Inc., Chicago, Estados Unidos). Para análise dos dados, foram obtidas distribuições absolutas e percentuais uni e bivariados. Na etapa de análise multivariada, recorreu-se à regressão logística binária. Foram criados dois modelos de regressão que adotaram como variáveis dependentes: conhecer o método e o uso incorreto do método, e admitindo as demais como independentes. Para todos os testes, foi estabelecido nível de significância igual a 0,05 para a rejeição de hipótese de nulidade.

O protocolo do projeto foi submetido ao Comitê de Ética em Pesquisa com seres humanos 
do Hospital Agamenon Magalhães, na cidade do Recife, sendo aprovado em julho de 2005.

\section{Resultados}

Dentre os 4.210 adolescentes, a maioria foi composta por moças, na faixa etária entre 17 e 19 anos, solteira, que se declarou de cor parda e residente na zona urbana da capital. As características sócio-demográficas e econômicas estão apresentadas na Tabela 1.

Tabela 1

Distribuição das variáveis sócio-demográficas e econômicas dos adolescentes do ensino médio da rede pública estadual de Pernambuco, Brasil.

\begin{tabular}{|c|c|c|}
\hline Variáveis & $\mathbf{n}$ & $\%$ \\
\hline \multicolumn{3}{|l|}{$\operatorname{Sexo}(n=4.210)$} \\
\hline Masculino & 1.693 & 40,2 \\
\hline Feminino & 2.517 & 59,8 \\
\hline \multicolumn{3}{|l|}{ Faixa etária (anos) [ $n=4.210]$} \\
\hline $14-16$ & 1.769 & 42,0 \\
\hline $17-19$ & 2.441 & 58,0 \\
\hline \multicolumn{3}{|l|}{ Estado civil $(n=4.153)$} \\
\hline Solteiro (a) & 3.920 & 94,4 \\
\hline Casado ou vivendo com companheiro(a) & 143 & 3,4 \\
\hline Viúvo ou separado & 90 & 2,2 \\
\hline \multicolumn{3}{|l|}{ Cor da pele $(n=4.210)$} \\
\hline Parda & 2.867 & 68,1 \\
\hline Branca & 1.057 & 25,1 \\
\hline Amarela & 157 & 3,7 \\
\hline Preta & 99 & 2,4 \\
\hline Indígena & 30 & 0,7 \\
\hline \multicolumn{3}{|l|}{ Ocupação ( $n=4.210)$} \\
\hline Estudante & 3.311 & 78,6 \\
\hline Estudante com outra ocupação & 899 & 21,4 \\
\hline \multicolumn{3}{|l|}{ Região geográfica $(n=4.210)$} \\
\hline Região metropolitana & 1.759 & 41,8 \\
\hline Zona da mata & 746 & 17,6 \\
\hline Agreste & 704 & 16,7 \\
\hline Sertão & 575 & 13,7 \\
\hline Sertão do São Francisco & 429 & 10,2 \\
\hline \multicolumn{3}{|l|}{ Local de residência $(n=4.183$ ) } \\
\hline Zona urbana & 3.302 & 78,9 \\
\hline Zona rural & 881 & 21,1 \\
\hline \multicolumn{3}{|l|}{ Posse de computador $(n=4.189)$} \\
\hline $\operatorname{Sim}$ & 447 & 10,7 \\
\hline Não & 3.742 & 89,3 \\
\hline \multicolumn{3}{|l|}{ Escolaridade materna $(n=3.954)$} \\
\hline lletrada & 674 & 17,0 \\
\hline Ensino Fundamental & 2.379 & 60,2 \\
\hline Ensino Médio & 730 & 18,5 \\
\hline Ensino Superior completo & 171 & 4,3 \\
\hline
\end{tabular}

Cerca de quatro em cada dez adolescentes relataram ter tido relação sexual na vida, sendo observada uma proporção significantemente maior entre os rapazes $(70,4 \%)$ em comparação às moças $(26,4 \% ; \mathrm{p}<0,001)$. Entre aqueles adolescentes que afirmaram já ter iniciado vida sexual, cerca da metade das moças afirmou primeira relação sexual na idade de 16 anos ou mais. Entre os rapazes, proporção semelhante afirmou que a primeira relação sexual ocorreu até os 14 anos de idade (Tabela 2).

Quanto ao número de parceiros sexuais na vida, $60,3 \%$ dos rapazes e $23,5 \%$ das moças afirmaram ter tido três parceiros ou mais $(\mathrm{p}<0,001)$. Dentre os adolescentes com vida sexual iniciada, a grande maioria relatou ter usado preservativo durante a última relação, não sendo observada diferença significante entre rapazes e moças (Tabela 2).

Independentemente de ter iniciado a vida sexual, a maioria afirmou conhecer o método contraceptivo de emergência, apesar de 35\% terem relatado que nunca receberam informações a respeito. Os principais agentes de informação foram os amigos (15,5\%), pais ou parentes $(14,6 \%)$, profissionais de saúde (14\%) e professores $(10,6 \%)$, enquanto a minoria afirmou ter recebido informações por meio de propaganda $(5,9 \%)$ e na farmácia $(4,5 \%)$, conforme os valores apresentados na Tabela 2 .

$\mathrm{Na}$ análise bivariada, observou-se associação significante entre o conhecimento do método contraceptivo de emergência e as variáveis: sexo, faixa etária, estado civil, morar com a família, local de residência, posse de computador, região geográfica, escolaridade materna, relação sexual e idade à primeira relação. Todavia, na análise de regressão ajustada, observou-se que as moças (5,03; IC95\%: 1,72-14,69) e os adolescentes que relataram ter tido relação sexual pelo menos uma vez na vida (1,52; IC95\%: 1,34$1,75)$ apresentam maior chance de conhecer o método (Tabela 3).

As variáveis que discriminaram a forma da utilização do método de contracepção de emergência foram: sexo, morar com a família, local de residência, região geográfica, uso do preservativo durante a última relação e informação sobre o método. Quando controlado para as variáveis de confusão, verificou-se que os adolescentes residentes do interior apresentam 1,68 (IC95\%: 1,09-2,25) vezes mais chance de usar incorretamente o método em comparação aos residentes da região metropolitana. Constatouse ainda um fator de proteção entre as moças $(0,29$; IC95\%: 0,15-0,54) e entre os adolescentes que afirmaram ter recebido informações sobre o método (0,78; IC95\%: 0,23-0,92 - Tabela 4). 
Distribuição das variáveis relativas ao comportamento sexual e contracepção de emergência, estratificada por sexo.

\begin{tabular}{|c|c|c|c|c|c|c|}
\hline \multirow[t]{2}{*}{ Variáveis } & \multicolumn{2}{|c|}{ Todos } & \multicolumn{2}{|c|}{ Rapazes } & \multicolumn{2}{|c|}{ Moças } \\
\hline & $\mathbf{n}$ & $\%$ & $\mathbf{n}$ & $\%$ & $\mathbf{n}$ & $\%$ \\
\hline \multicolumn{7}{|l|}{ Relação sexual $(n=4.185)$} \\
\hline $\operatorname{Sim}$ & 1.844 & 44,1 & 1.184 & 70,4 & 660 & 26,4 \\
\hline Não & 2.341 & 55,9 & 497 & 29,6 & 1.844 & 73,6 \\
\hline \multicolumn{7}{|c|}{ Idade da primeira relação sexual (anos) [n $=1.834]$ * } \\
\hline Menos de 12 & 104 & 5,7 & 99 & 8,3 & 5 & 0,8 \\
\hline 12 & 147 & 8,0 & 132 & 11,1 & 15 & 2,3 \\
\hline 13 & 211 & 11,5 & 170 & 14,3 & 41 & 6,4 \\
\hline 14 & 355 & 19,4 & 241 & 20,3 & 114 & 17,7 \\
\hline 15 & 448 & 24,4 & 290 & 24,4 & 158 & 24,5 \\
\hline 16 ou mais & 569 & 31,0 & 257 & 21,6 & 312 & 48,4 \\
\hline \multicolumn{7}{|l|}{ Número de parceiros sexuais na vida $(n=1.843)$ * } \\
\hline 1 & 635 & 34,5 & 249 & 20,9 & 386 & 59,5 \\
\hline 2 & 336 & 18,2 & 225 & 18,8 & 111 & 17,1 \\
\hline 3 & 248 & 13,5 & 186 & 15,6 & 62 & 9,6 \\
\hline 4 & 158 & 8,6 & 125 & 10,5 & 33 & 5,1 \\
\hline 5 & 121 & 6,6 & 97 & 8,1 & 24 & 3,7 \\
\hline 6 ou mais & 345 & 18,7 & 312 & 26,1 & 33 & 5,1 \\
\hline \multicolumn{7}{|l|}{ Uso de preservativos na última relação $(n=1.848)$ * } \\
\hline Não & 515 & 27,9 & 278 & 23,3 & 237 & 36,2 \\
\hline Sim & 1.333 & 72,1 & 916 & 76,7 & 417 & 63,8 \\
\hline \multicolumn{7}{|l|}{ Conhecimento sobre o contraceptivo $(n=4.170)$} \\
\hline Não tenho relações sexuais e não conheço & 1.091 & 26,2 & 294 & 17,6 & 797 & 31,9 \\
\hline Tenho relações sexuais e não conheço & 518 & 12,4 & 414 & 24,8 & 104 & 4,2 \\
\hline Não tenho relações sexuais e conheço & 1.453 & 34,4 & 344 & 20,6 & 1.089 & 43,6 \\
\hline Tenho relações sexuais e conheço & 1.128 & 27,1 & 619 & 37,0 & 509 & 20,4 \\
\hline \multicolumn{7}{|l|}{ Agentes de informação $(n=4.131)$} \\
\hline Nenhum & 1446 & 35,0 & 621 & 37,3 & 825 & 33,4 \\
\hline Pais ou parentes & 603 & 14,6 & 215 & 12,9 & 388 & 15,7 \\
\hline Amigos & 639 & 15,5 & 254 & 15,3 & 385 & 15,6 \\
\hline Profissionais de saúde & 578 & 14,0 & 231 & 13,9 & 347 & 14,1 \\
\hline Propagandas & 245 & 5,9 & 96 & 5,8 & 149 & 6,0 \\
\hline Farmácias & 184 & 4,5 & 102 & 6,1 & 82 & 3,3 \\
\hline Professores & 436 & 10,6 & 145 & 8,7 & 291 & 11,8 \\
\hline \multicolumn{7}{|c|}{ Freqüência de uso nos últimos 12 meses (vezes) [n $=1.764]$ * } \\
\hline Nunca usei (ou minha parceira usou) & 1.283 & 72,7 & 823 & 72,9 & 460 & 72,4 \\
\hline $1-2$ & 323 & 18,3 & 212 & 18,8 & 111 & 17,5 \\
\hline 3 ou mais & 148 & 9,0 & 84 & 8,3 & 64 & 10,0 \\
\hline \multicolumn{7}{|l|}{ Forma de uso $(n=542) * \star$} \\
\hline Até 72 horas pós-coito & 120 & 22,1 & 53 & 15,9 & 67 & 32,2 \\
\hline Quando falta a menstruação & 80 & 14,7 & 45 & 13,5 & 35 & 16,9 \\
\hline Antes do ato sexual & 254 & 46,9 & 195 & 58,5 & 59 & 28,4 \\
\hline Após as 72 horas pós-coito & 88 & 16,3 & 41 & 12,1 & 47 & 22,5 \\
\hline
\end{tabular}

* Incluídos apenas os adolescentes que relataram ter tido relação sexual;

** Incluídos apenas os adolescentes que relataram ter usado método contraceptivo. 
Análise bivariada e odds ratio (OR) brutas e ajustadas entre o conhecimento do método e as variáveis sócio-demográficas e relacionadas ao comportamento sexual.

\begin{tabular}{|c|c|c|c|c|c|c|c|c|}
\hline \multirow[t]{2}{*}{ Variáveis } & \multicolumn{2}{|c|}{ Não conhece } & \multicolumn{2}{|c|}{ Conhece } & \multicolumn{4}{|c|}{ Desfecho: conhecimento do método } \\
\hline & $\mathbf{n}$ & $\%$ & $\mathbf{n}$ & $\%$ & $\begin{array}{l}\text { OR bruto } \\
\text { (IC95\%) }\end{array}$ & $\begin{array}{l}\text { Valor } \\
\text { de } p\end{array}$ & $\begin{array}{l}\text { OR ajustado } \\
\text { (IC95\%)* }\end{array}$ & $\begin{array}{l}\text { Valor } \\
\text { de p }\end{array}$ \\
\hline \multicolumn{9}{|l|}{ Sexo } \\
\hline Rapazes & 708 & 42,4 & 963 & 57,6 & 1,00 & & 1,00 & \\
\hline Mocas & 901 & 36,1 & 1.598 & 63,9 & $1,30(1,15-1,48)$ & 0,00 & $5,03(1,72-14,69)$ & 0,00 \\
\hline \multicolumn{9}{|l|}{ Faixa etária (anos) } \\
\hline $14-16$ & 756 & 43,0 & 1.001 & 57,0 & 1,00 & & 1,00 & \\
\hline $17-19$ & 857 & 35,4 & 1.565 & 64,6 & $1,38(1,21-1,56)$ & 0,00 & $1,18(0,59-2,37)$ & 0,67 \\
\hline \multicolumn{9}{|l|}{ Estado civil } \\
\hline Solteiro (a) & 1.549 & 39,5 & 2.371 & 60,5 & 1,00 & & 1,00 & \\
\hline Casado(a)/parceiro(a) & 50 & 21,5 & 183 & 78,5 & $2,40(1,74-3,29)$ & 0,00 & $2,58(0,27-24,26)$ & 0,40 \\
\hline \multicolumn{9}{|l|}{ Morar com a família } \\
\hline Sim & 1.097 & 41,8 & 1.527 & 60,2 & 1,00 & & 1,00 & \\
\hline Não & 495 & 32,9 & 1.010 & 67,1 & $1,46(1,28-1,67)$ & 0,00 & $1,32(0,64-2,75)$ & 0,49 \\
\hline \multicolumn{9}{|l|}{ Cor da pele } \\
\hline Branca & 385 & 36,7 & 663 & 63,3 & 1,00 & & 1,00 & \\
\hline Não branca & 1.223 & 39,2 & 1.896 & 60,8 & $0,90(0,78-1,04)$ & 0,15 & $0,72(0,35-1,51)$ & 0,40 \\
\hline \multicolumn{9}{|l|}{ Ocupação } \\
\hline Não & 1.254 & 38,4 & 2.008 & 61,6 & 1,00 & & 1,00 & \\
\hline $\operatorname{Sim}$ & 344 & 38,7 & 546 & 61,3 & $0,99(0,85-1,15)$ & 0,91 & $1,38(0,70-2,72)$ & 0,35 \\
\hline \multicolumn{9}{|l|}{ Local de residência } \\
\hline Urbana & 1.152 & 35,1 & 2127 & 64,9 & 1,00 & & 1,00 & \\
\hline Rural & 456 & 52,2 & 418 & 47,8 & $0,49(0,42-0,57)$ & 0,00 & $0,65(0,31-1,38)$ & 0,26 \\
\hline \multicolumn{9}{|l|}{ Computador } \\
\hline Sim & 119 & 27,0 & 322 & 73,0 & 1,00 & & 1,00 & \\
\hline Não & 1.489 & 40,1 & 2.228 & 59,9 & $0,55(0,44-0,69)$ & 0,00 & $0,41(0,13-1,36)$ & 0,13 \\
\hline \multicolumn{9}{|l|}{ Região Geográfica } \\
\hline Região metropolitana & 537 & 30,7 & 1.210 & 69,3 & 1,00 & & 1,00 & \\
\hline Interior & 1.076 & 44,2 & 1.356 & 55,8 & $0,56(0,49-0,63)$ & 0,00 & $0,32(0,14-0,70)$ & 0,05 \\
\hline \multicolumn{9}{|l|}{ Escolaridade da mãe (anos) } \\
\hline$\leq 8$ & 1.153 & 40,5 & 1.693 & 59,5 & 1,00 & & 1,00 & \\
\hline 9 ou mais & 335 & 31,0 & 747 & 69,0 & $1,51(1,30-1,76)$ & 0,00 & $0,88(0,42-1,86)$ & 0,74 \\
\hline \multicolumn{9}{|l|}{ Já teve relação sexual } \\
\hline Não & 1.025 & 43,9 & 1.394 & 56,1 & 1,00 & & 1,00 & \\
\hline Sim & 587 & 32,0 & 1.248 & 68,0 & $1,66(1,46-1,89)$ & 0,00 & $1,52(1,34-1,75)$ & 0,00 \\
\hline \multicolumn{9}{|c|}{ Idade à primeira relação (anos) ** } \\
\hline$\leq 14$ & 267 & 32,9 & 545 & 67,1 & 1,00 & & 1,00 & \\
\hline 15 ou mais & 314 & 31,0 & 700 & 69,0 & $1,04(0,95-1,49)$ & 0,38 & $1,05(0,94-1,50)$ & 0,40 \\
\hline \multicolumn{9}{|l|}{ Número de parceiros ** } \\
\hline $1-2$ & 310 & 32,3 & 654 & 67,8 & 1,00 & & 1,00 & \\
\hline$\geq 3$ & 271 & 31,1 & 599 & 68,9 & $1,04(0,86-1,27)$ & 0,64 & $1,06(0,82-1,33)$ & 0,68 \\
\hline \multicolumn{9}{|c|}{ Preservativo na última relação ** } \\
\hline Sim & 440 & 33,1 & 890 & 66,9 & 1,00 & & 1,00 & \\
\hline Não & 150 & 29,3 & 362 & 70,7 & $1,19(0,95-1,49)$ & 0,11 & $1,20(0,90-1,52)$ & 0,24 \\
\hline
\end{tabular}

* Ajustado para todas as sócio-demográficas e relacionadas ao comportamento sexual;

** Incluídos apenas os adolescentes que relataram ter tido relação sexual. 


\section{Discussão}

Nesse estudo, a maioria dos entrevistados foi feminina. Moças residentes na região metropolitana e urbana, de cor parda, corroborando a tendência relatada no documento da Comissão Nacional de População e Desenvolvimento e em outros estudos 22,23. Esta distribuição revela uma importância social, com reflexos sobre a vida reprodutiva, havendo a possibilidade de que alguns fatores, como, por exemplo, a necessidade de trabalhar para compor a renda familiar e a gravidez inoportuna, tenham sido responsáveis pela diminuição da presença masculina na escola 24 .

$O$ resultado da presente pesquisa, quanto ao relato feito por metade dos sujeitos de nunca ter tido relações sexuais, parece não condizer com o que é veiculado pela mídia referente a vigorar no país a representação de uma cultura sexualmente muito aberta. Heilborn 25 , com base em um inquérito domiciliar realizado em três capitais de regiões geográficas brasileiras distintas, comprova que a idéia hegemônica da sexualidade brasileira erotizada, referida internacionalmente, não passa de um mito.

A iniciação sexual precoce entre adolescentes gera preocupação em decorrência da freqüente associação desse comportamento com desconhecimento sobre anticoncepção e saúde reprodutiva. Aliada à atividade sexual precoce e ao desconhecimento das questões da sexualidade, tem sido comprovada pouca participação da família, da escola e dos serviços de saúde no processo educativo de adolescentes, sendo os próprios colegas - também adolescentes - sua principal fonte de informações.

A constatação de que $44,1 \%$ dos adolescentes pesquisados relataram ter iniciado vida sexual, sendo 5,7\%, menores de 12 anos de idade e $30,9 \%$, na faixa etária dos 13 aos 14 anos, reflete uma situação crítica pela possibilidade de serem surpreendidos por uma gravidez inoportuna, dada a fase de vida em que se encontram, caracterizada pela tendência de correr riscos de forma inconseqüente. Nesse processo, a mídia tem desempenhado papel de destaque em razão da ampla e massificada divulgação de informações a respeito da atividade e do comportamento sexual nos diferentes grupos sociais, mas o faz sem estabelecimento de critérios de causa e efeito. É esperado que, nesse patamar de faixa etária, o jovem não tenha alcançado os níveis de complexidade e de aprendizagem necessários para a tomada de decisão no exercício da vida sexual 26.

Por outro lado, há registros de conflitos com os pais por considerarem que os professores estariam promovendo a sexualidade dos alunos, ao incentivarem a interação entre pares. A socialização dos jovens no campo da sexualidade se dá especialmente entre os pares, o que pode representar tanto um fator de proteção quanto de risco 23. Os adolescentes, que afirmaram conviver com amigos que preenchem tal papel, apresentam quase o dobro de chances de usar o condom em suas relações sexuais, quando comparados àqueles que não conversam sobre vida sexual. No entanto os pares também podem influir negativamente, ao estimularem o comportamento de risco, a depender do que prevalece no grupo.

Considerando que a influência dos pares aumenta com a idade do adolescente, amigos mais velhos têm importância na orientação dos mais jovens sobre a necessidade de proteção e o significado maior de seu estímulo, quando se trata da iniciação sexual, particularmente no que se refere ao uso de preservativo, quando da primeira relação, que pode consolidar seu uso persistente 27 .

O depoimento da maioria dos jovens pesquisados apontou para a construção de uma atitude favorável quanto ao uso do condom, porém houve informação de relacionamento sexual desprotegido; procedimento que os torna vulneráveis às DST e a gestações precoces ou inesperadas 28 .

Quanto ao número de parceiros sexuais, os percentuais foram maiores que os referidos por Abramovay 23 (46,5\%, 9,1\% e 35,2\%, respectivamente, para rapazes, moças e total amostral na faixa etária de 15 a 19 anos, para dois a cinco parceiros sexuais na vida). Considerando a intensidade da vida sexual por gênero e ciclos etários na geração jovem, as autoras referem que em todas as faixas etárias, entre 10 e 24 anos, há divisões sexuais nítidas, aparecendo as moças com mais baixas proporções quando se considera o número de parceiros. O comum, na maioria das capitais brasileiras, é que crianças e jovens de 10 a 14 anos tendam a assumir que tiveram relação sexual com uma pessoa apenas, no último ano, atingindo percentuais que variam de $91 \%$, em Florianópolis, a 57\%, em Cuiabá, diferente do constatado em Fortaleza, onde $32 \%$ desses jovens afirmaram ter tido relações sexuais com duas a cinco pessoas, no último ano ${ }^{23}$.

Em relação à contracepção de emergência, uma minoria de adolescentes informou não ter tido qualquer orientação, mas a maioria referiu conhecer, incluindo usuários e não usuários. No entanto foi preocupante constatar que houve predomínio do uso incorreto desse método contraceptivo. Figueiredo \& Andalaft Neto 29, pesquisando estudantes de Ensino Médio de escolas estaduais de São Paulo, ao identificarem conhecimento da contracepção de emergência por $56 \%$ das pesquisadas, entenderam que ainda 
Análise bivariada e odds ratio (OR) brutas e ajustadas entre o uso incorreto do contraceptivo de emergência e as variáveis sócio-demográficas, relacionadas ao comportamento sexual e a contracepção de emergência.

\begin{tabular}{|c|c|c|c|c|c|c|c|c|}
\hline \multirow[t]{2}{*}{ Variáveis * } & \multicolumn{2}{|c|}{ Correta } & \multicolumn{2}{|c|}{ Incorreta } & \multicolumn{4}{|c|}{ Desfecho: Uso incorreto } \\
\hline & $\mathrm{n}$ & $\%$ & $\mathrm{n}$ & $\%$ & $\begin{array}{l}\text { OR bruto } \\
\text { (IC95\%) }\end{array}$ & $\begin{array}{l}\text { Valor } \\
\text { de } p\end{array}$ & $\begin{array}{l}\text { OR ajustado ** } \\
\text { (IC95\%) }\end{array}$ & $\begin{array}{l}\text { Valor } \\
\text { de } p\end{array}$ \\
\hline \multicolumn{9}{|l|}{ Sexo } \\
\hline Masculino & 53 & 15,9 & 281 & 84,1 & 1,00 & & 1,00 & \\
\hline Feminino & 67 & 32,2 & 141 & 67,8 & $0,54(0,40-0,72)$ & 0,00 & $0,29(0,15-0,54)$ & 0,00 \\
\hline \multicolumn{9}{|l|}{ Faixa etária (anos) } \\
\hline $14-16$ & 30 & 17,9 & 138 & 82,1 & 1,00 & & 1,00 & \\
\hline $17-19$ & 90 & 24,0 & 284 & 76,0 & $0,69(0,43-1,09)$ & 0,11 & $0,65(0,35-1,20)$ & 0,17 \\
\hline \multicolumn{9}{|l|}{ Estado civil } \\
\hline Solteiro(a) & 104 & 21,8 & 373 & 78,2 & 1,00 & & 1,00 & \\
\hline Casado(a)/parceiro(a) & 16 & 26,6 & 45 & 73,8 & $1,03(0,72-1,48)$ & 0,85 & $2,03(0,90-4,61)$ & 0,08 \\
\hline \multicolumn{9}{|l|}{ Morar com a família } \\
\hline Sim & 62 & 18,8 & 268 & 81,2 & 1,00 & & 1,00 & \\
\hline Não & 53 & 26,4 & 148 & 73,6 & $0,72(0,53-0,98)$ & 0,03 & $1,01(0,59-1,76)$ & 0,96 \\
\hline \multicolumn{9}{|l|}{ Cor da pele } \\
\hline Branca & 36 & 27,1 & 97 & 72,9 & 1,00 & & 1,00 & \\
\hline Não branca & 83 & 20,4 & 323 & 79,6 & $1,18(0,84-1,65)$ & 0,32 & $1,67(0,96-2,90)$ & 0,07 \\
\hline \multicolumn{9}{|l|}{ Ocupação } \\
\hline Não & 91 & 23,9 & 290 & 76,1 & 1,00 & & 1,00 & \\
\hline $\operatorname{Sim}$ & 27 & 17,3 & 129 & 82,7 & $1,24(0,91-0,70)$ & 0,16 & $1,25(0,70-2,23)$ & 0,44 \\
\hline \multicolumn{9}{|l|}{ Local de residência } \\
\hline Urbana & 101 & 22,9 & 341 & 77,1 & 1,00 & & 1,00 & \\
\hline Rural & 17 & 17,9 & 78 & 82,1 & $1,60(1,06-2,41)$ & 0,02 & $1,04(0,51-2,10)$ & 0,91 \\
\hline \multicolumn{9}{|l|}{ Computador } \\
\hline Sim & 17 & 24,3 & 53 & 17,7 & 1,00 & & 1,00 & \\
\hline Não & 102 & 21,8 & 366 & 78,2 & $1,27(0,82-1,96)$ & 0,27 & $1,67(0,82-3,40)$ & 0,15 \\
\hline \multicolumn{9}{|l|}{ Região geográfica } \\
\hline Região metropolitana & 63 & 26,8 & 172 & 73,2 & 1,00 & & 1,00 & \\
\hline Interior & 57 & 18,5 & 251 & 81,5 & $1,54(1,14-2,07)$ & 0,00 & $1,68(1,09-2,25)$ & 0,00 \\
\hline \multicolumn{9}{|c|}{ Escolaridade da mãe (anos) } \\
\hline$\leq 8$ & 85 & 23,2 & 281 & 76,8 & 1,00 & & 1,00 & \\
\hline 9 ou mais & 30 & 20,4 & 117 & 79,6 & $0,93(0,65-1,34)$ & 0,72 & $1,18(0,66-2,13)$ & 0,56 \\
\hline \multicolumn{9}{|c|}{ Idade da primeira relação(anos) } \\
\hline$\leq 14$ & 55 & 24,1 & 173 & 75,9 & 1,00 & & 1,00 & \\
\hline 15 ou mais & 64 & 23,1 & 213 & 76,9 & $0,94(0,69-1,27)$ & 0,69 & $1,45(0,81-2,60)$ & 0,21 \\
\hline \multicolumn{9}{|l|}{ Número de parceiros } \\
\hline $1-2$ & 61 & 24,7 & 186 & 75,3 & 1,00 & & 1,00 & \\
\hline$\geq 3$ & 57 & 22,0 & 202 & 78,0 & $0,88(0,65-1,19)$ & 0,40 & $0,91(0,50-1,69)$ & 0,78 \\
\hline \multicolumn{9}{|c|}{ Preservativo na última relação } \\
\hline Sim & 79 & 20,5 & 306 & 79,5 & 1,00 & & 1,00 & \\
\hline Não & 40 & 33,3 & 80 & 66,7 & $0,68(0,51-0,95)$ & 0,02 & $1,11(0,63-1,96)$ & 0,71 \\
\hline \multicolumn{9}{|c|}{ Informação sobre contraceptivo } \\
\hline Nenhum & 5 & 9,1 & 50 & 90,9 & 1,00 & & 1,00 & \\
\hline $\operatorname{Sim}$ & 114 & 24,1 & 352 & 75,5 & $0,50(0,28-0,88)$ & 0,01 & $0,78(0,23-0,92)$ & 0,04 \\
\hline \multicolumn{9}{|c|}{ Uso do contraceptivo (vezes) } \\
\hline $1-3$ & 80 & 26,3 & 224 & 73,7 & 1,00 & & 1,00 & \\
\hline$\geq 4$ & 27 & 25,7 & 78 & 74,3 & $1,18(0,82-1,71)$ & 0,36 & $1,25(0,94-1,98)$ & 0,15 \\
\hline
\end{tabular}

* Incluídos apenas os que relataram o uso do contraceptivo de emergência;

** Ajustado para as variáveis sócio-demográficas, relacionadas ao comportamento sexual e às variáveis sobre o contraceptivo de emergência. 
uma parte significativa dos jovens só conseguiu atingir o primeiro estágio da prevenção, ou seja, o uso de métodos contraceptivos que evitam gravidez, sem a preocupação de evitar DST/AIDS.

A idade precoce de iniciação da vida sexual dos adolescentes revela-se cada vez mais como um forte determinante do comportamento sexual e reprodutivo. Por esse motivo, a importância da contracepção de emergência respalda-se na idéia de que muitas gestações inoportunas ou indesejadas podem resultar em abortamentos nem sempre seguros, com sérios riscos à saúde sexual e reprodutiva. Embora sejam realizadas palestras nos serviços de saúde, como atividades planejadas ou mesmo pontuais, estas foram alvo de críticas por parte dos adolescentes pesquisados, que as consideram monótonas, fornecendo informações distantes de suas vivências e de suas emoções, assim sendo desinteressantes ou não apropriadas, situação que reduz sua efetividade em atingir o objetivo da disseminação de informações sobre métodos contraceptivos e de prevenção das DST.

Hardy et al. 18, investigando a contracepção de emergência, identificaram que os participantes manifestaram-se francamente favoráveis à provisão e disseminação dessa informação e a seu uso no Brasil. Consideraram que não existem barreiras significativas a sua aceitação pela sociedade, em geral, e que seria mais apropriado adotar-se a estratégia de inseri-la em programas abrangentes de saúde reprodutiva; como também o método deveria ser oferecido como mais uma alternativa contraceptiva, em meio às demais, enfatizando o que se deve admitir como emergência, além de apontarem como essencial que os profissionais de saúde sejam capacitados para prover a informação e o método. Esta parece ser a condição indispensável para que os serviços de saúde possam contribuir efetivamente na redução da vulnerabilidade às DST e às gestações precoces, nesse grupo populacional.

O desconhecimento da contracepção de emergência tem sido referido por diversos autores. Nunes ${ }^{30}$, entrevistando 419 adolescentes de ensino médio, em Portugal, em 2003, identificou que $94,3 \%$ sabiam da existência desse método, mas apenas $15,8 \%$ eram capazes de identificar a forma correta de uso. Costa et al. 15, investigando 135 adolescentes do sexo feminino do ensino médio de escolas públicas da cidade do Rio de Janeiro, referiram que $32,6 \%$ haviam feito uso da contracepção de emergência, mas $43,2 \%$ delas avaliaram como insuficientes as informações de que dispunham para sua utilização. Diaz et al. 2, investigando 75 adolescentes residentes no Brasil, no Chile e no México, classificaram como incompleto seu conhecimento sobre contracep- ção de emergência. Romo et al. 5, investigando 69 adolescentes latino-americanas residentes no Texas, Estados Unidos, verificaram que 24,6\% tinham informações sobre contracepção de emergência, mas apenas $12 \%$ delas conheciam o uso correto.

Nunes 30, em Portugal, ao discutir a falta de conhecimento sobre contracepção de emergência, sugeriu que a informação recebida era incompleta ou inadequada e atribuiu o fato ao agente de informação. Tal como se verificou na presente pesquisa, diferente do ideal, os profissionais de saúde e os professores não foram as principais fontes de informação.

Diversos estudos 11,12,31,32 têm referido temor por parte dos profissionais de saúde da divulgação da contracepção de emergência, especialmente para adolescentes, pela possibilidade do mau uso do método e de sua priorização, em detrimento da anticoncepção de rotina. Considerando os dados da presente pesquisa, foi possível atribuir esse mau uso à falta de informação ou mesmo à informação incorreta, visto que $9 \%$ dos adolescentes pesquisados referiram uso freqüente e $77,9 \%$, incorreto.

Os adolescentes recorreram aos amigos, provavelmente não preparados para isso e, por conseguinte, podem ter recebido informações até mesmo deformadas, o que até certo ponto era de se esperar. O fato preocupante que os resultados da presente pesquisa apontam é a baixa participação dos profissionais de saúde como agentes de informação. Nunes ${ }^{30}$, ao encontrar resultados semelhantes, oferece duas explicações que também parecem pertinentes na presente pesquisa. Em primeiro lugar, a possibilidade do pudor do adolescente em buscar nos adultos uma fonte de informação desse tipo, pelo medo da interpretação que o agente de informação possa ter a seu respeito e pela necessidade de não revelar detalhes de sua vida sexual. Por outro lado, questiona o despreparo dos profissionais de saúde para abordar a contracepção de emergência 15, pelos motivos referidos anteriormente.

A respeito do uso da contracepção de emergência, Wynn \& Trussell 33 manifestam uma opinião diferente ao questionarem se a ênfase da contracepção de emergência não é marcada por uma ambigüidade científica, ao preconizar uma ideologia da sexualidade, enquanto prática despida de relacionamento afetivo, tendo, como conseqüência, a gravidez como evento inoportuno. Argumentam que os mais importantes objetivos da saúde pública são preconizar um comportamento psicologicamente sadio com conseqüências potencialmente benéficas em longo prazo, às quais se contrapõe um relacionamento sexual inoportuno ou inadequado, quando pre- 
coce, mais do que a contracepção inoportuna ou o abortamento. Isso infringe o direito desse equilíbrio psíquico, independentemente do credo religioso. Os autores 33 consideram que os debates sobre contracepção de emergência devem, sobretudo, enfatizar o que seja emergência, apontar os riscos a ela inerentes e priorizar todas as ações de educação sexual, como processo de formação da decisão responsável de ter relação sexual e não de resolver o exercício sexual realizado sem proteção contraceptiva, com riscos futuros à saúde e, desse modo, contrários aos interesses da saúde pública. Isso enseja início de vida sexual precoce e intercurso com diversos parceiros, tal como observado no presente estudo.

Ao analisar os fatores intervenientes sobre o conhecimento da contracepção de emergência e o uso correto desse método pelos adolescentes pesquisados, a identificação de que o conhecimento teve predomínio significante entre as moças da Região Metropolitana que tinham informação sobre contraceptivo pareceu indicar uma influência sócio-econômica. Significa dizer que o segmento social com características diferentes destas, esteve mais vulnerável; é nele que se concentram a maior fertilidade e a maior freqüência de gravidez inoportuna; também é nele, portanto, que se devem concentrar os maiores esforços de educação sexual e saúde reprodutiva.

Nunes 30 e Wynn \& Trussell 33 consideram que, numa sociedade em que a relação médico/ paciente vem sendo desgastada, assim como se reduz o tempo disponível para o contato entre pais e filhos por contingências sócio-econômicas ou mesmo por um afastamento decorrente de conflito de gerações, parece mais fácil oferecer ou recomendar aos adolescentes a leitura de textos diversos do que discutir com eles, diretamente, certos assuntos. Este tipo de conduta da família e da escola favorece a falta de informa- ção, assim como a deformação do conhecimento, inviabilizando uma discussão que poderia ser muito rica e para a qual não faltam participantes interessados para compreender a sexualidade e não apenas utilizar o sexo. Sem dúvida, a contracepção de emergência deve ser disponibilizada em socorro das mulheres que se vêem vítimas de situações que lhes infrinjam sofrimento, nem sempre decorrente de suas decisões.

O uso de contraceptivos, independentemente do tipo, pressupõe livre escolha, espaços democráticos de planejamento, avaliação e troca de experiências dos próprios adolescentes para incentivar sua participação nas ações de promoção de saúde. Por esse motivo, uma abordagem holística das especificidades individuais e culturais dos adolescentes constitui-se em direito de cidadania 34 . Somente quando for possível vincular teoria e prática para melhor intervir em todas as dimensões da sexualidade, no sentido de assegurar qualidade de vida aos cidadãos, ao invés de restringir as ações de saúde reprodutiva ao campo de atuação na gestação indesejável ou inoportuna, reconhecendo as interfaces e as conexões, será possível contribuir efetivamente para relações sexuais construtivas.

Ao constatar que, dentre os adolescentes que iniciaram a vida sexual, predominaram a idade maior que 15 anos, um número de três ou mais parceiros, a obtenção de informação predominantemente com os amigos e o emprego da contracepção de emergência de forma incorreta, concluiu-se haver a necessidade de desenvolver programas de educação sexual e reprodutiva efetivamente voltados para os adolescentes, numa linguagem mais próxima à sua realidade, para transformá-los em parceiros da adoção de condutas preventivas, uma vez que os amigos tiveram um papel preponderante na informação. 


\section{Resumo}

O objetivo deste estudo foi investigar o conhecimento e o uso do contraceptivo de emergência em 4.210 adolescentes (14-19 anos) da rede pública estadual de Pernambuco, Brasil. As informações foram coletadas pelo questionário Global School-Based Student Health Survey, previamente validado. Foi investigado o conhecimento, a freqüência e forma de uso do contraceptivo de emergência. As variáveis independentes foram divididas em sócio-demográficas e relacionadas ao comportamento sexual. A maioria dos adolescentes relatou conhecer e já ter recebido informações sobre o método, entretanto dentre os que já utilizaram apenas $22,1 \%$ o fez de forma correta. A análise ajustada de regressão evidenciou uma maior chance de conhecer o método entre as moças $(O R=5,03 ;$ IC95\%: 1,72-14,69) $e$ entre aqueles que já tiveram relação sexual $(\mathrm{OR}=$ 1,52; IC95\%: 1,34-1,75), enquanto os residentes do interior possuem $68 \%$ menos chance. Em relação ao uso, os residentes do interior apresentam 1,68 (OR; IC95\%: 1,09-2,25) mais chance de uso incorreto, enquanto as moças apresentam $71 \%$ menos chance. São necessárias ações de educação sexual e reprodutiva, sobretudo entre os rapazes e adolescentes do interior.

Anticoncepção; Adolescente; Comportamento Sexual

\section{Referências}

1. World Health Organization. Married adolescents: no place of safety. Geneva: World Health Organization; 2006. (WHO Library Cataloguing-in-Publication Data)

2. Díaz S, Hardy E, Alvarado G, Ezcurra E. Acceptability of emergency contraception in Brazil, Chile and México. 1 - Perceptions of emergency oral contraceptive. Cad Saúde Pública 2003; 19:1507-17.

3. Gold MA, Wolford JE, Smith KA, Parker AM. The effects of advance provision of emergency contraception on adolescent womens' sexual and contraceptive behaviors. J Pediatr Adolesc Gynecol 2004; 17:87-96

4. Guimarães AMDN, Vieira MJ, Palmeira JA. Informações dos adolescentes sobre métodos anticoncepcionais. Rev Latinoam Enferm 2003; 11:293-8.

5. Romo LF, Berenson AB, Wu ZH. The role of misconceptions on latino womens' acceptance of emergency contraceptive pills. Contraception 2004; 69:227-35.

\section{Colaboradores}

M. S. P. Araújo foi responsável pelo levantamento dos dados e redação deste artigo. L. O. B. F. Costa contribuiu com críticas para a construção da estrutura de idéias. Ambas as autoras colaboraram com a versão final do manuscrito.
6. Almeida MCC, Aquino EML, Gaffikin L, Magnani RJ. Uso de contracepção por adolescentes de escolas públicas na Bahia. Rev Saúde Pública 2003; 37:566-75.

7. Borges ALV. Adolescência e vida sexual: análise do início da vida sexual de adolescentes residentes na zona leste do município de São Paulo [Tese de Doutorado]. São Paulo: Faculdade de Saúde Pública, Universidade de São Paulo; 2004.

8. Fernandes MFM. Mulher, família e reprodução: um estudo de caso sobre planejamento familiar em periferia do Recife, Pernambuco, Brasil. Cad Saúde Pública 2003; 19 Suppl 2:S253-61.

9. Harper CC, Minnis AM, Padian NS. Sexual partners and use of emergency contraception. Am J Obstet Gynecol 2003; 189:1093-9. 
10. Pirotta KCM. Não há guarda-chuva contra o amor: estudo do comportamento reprodutivo e do seu universo simbólico entre jovens universitários da USP [Tese de Doutorado]. São Paulo: Faculdade de Saúde Pública, Universidade de São Paulo; 2002.

11. Churchill D. The growing pains of adolescent health research in general practice. Prim Health Care Res Dev 2003; 4:277-8.

12. Queiroz A. Novas tecnologias reprodutivas: uma oferta de possibilidades contraditórias para as mulheres. Revista Estudos Feministas 2002; 10: 239-41.

13. Diniz D, Guilhem, D. Bioética feminista: o resgate político do conceito de vulnerabilidade. Revista Bioética 2000; 2:1-2.

14. Tripp J, Viner R. Sexual health, contraception, and teenage pregnancy. BMJ 2005; 330:590-3.

15. Costa KBS, Carvalho ACG, Kivia BS, Mendes SHFM, Soares A. Contracepção de adolescentes do Rio de Janeiro. Revista da SOGIA-BR 2006; 7:3-6.

16. Carret MLV, Fassa AG, Silveira DS, Bertoldi AD, Hallal P. Sintomas de doenças sexualmente transmissíveis em adultos: prevalência e fatores de risco. Rev Saúde Pública 2004; 38:76-84.

17. Sundby J, Svanemyr J, Maehre T. Avoiding unwanted pregnancy: the role of communication, information and knowledge in the use of contraception among young Norwegian women. Patient Educ Couns 1999; 38:11-9.

18. Hardy E, Duarte GA, Osis MJD, Arce XE, Possan M. Emergency contraception in Brazil: facilitators and barriers. Cad Saúde Pública 2001; 17:1031-5.

19. Secretaria de Estado da Educação e Cultura. Censo escolar. Número de escolas por município, dependência administrativa e tipo de ensino. Recife: Secretaria de Estado da Educação e Cultura; 2005.

20. Luiz RR, Magnanini MF. A lógica da determinação do tamanho da amostra em investigações epidemiológicas. Cad Saúde Coletiva 2000; 2:9-28.

21. Hallal PC, Victora CG, Wells JC, Lima RC. Physical inactivity: prevalence and associated variables in brasilian adults. Med Sci Sports Exerc 2003; 35:1894-900.

22. Leite, APL. Sexualidade na adolescência: conhecimentos, atitudes e práticas dos adolescentes estudantes do Município de Maceió [Dissertação de Mestrado]. Recife: Faculdade de Ciências Médicas, Universidade de Pernambuco; 2000.

23. Abramovay M. Juventudes e sexualidade. Brasília: Organização das Nações Unidas para a Educação, a Ciência e a Cultura; 2004
24. Catharino TR, Giffin K. Gravidez e adolescência - investigação de um problema moderno. In: XIII Encontro da Associação Brasileira de Estudos Populacionais. Ouro Preto: Associação Brasileira de Estudos Populacionais; 2002. p. 1-20.

25. Heilborn ML. Entre as tramas da sexualidade brasileira. Revista Estudos Feministas 2006; 14:336.

26. Paraguassú ALCB, Costa MCO, Sobrinho CLN, Patel BN, Freitas JT, Araújo, FPO. Situação sócio-demográfica e de saúde reprodutiva pré e pós-gestacional de adolescentes, Feira de Santana, Bahia, Brasil. Ciênc Saúde Coletiva 2005; 10:373-80.

27. Lefkowitz ES, Boone TL, Shearer CL. Communication with best friends about sex-related topics during emerging adulthood. J Youth Adolesc 2004; 33:339-51.

28. Moreira MM, Juarez F. Comportamento sexual de adolescentes do sexo masculino de baixa renda: a comunidade de Chão de Estrelas em Recife, Pernambuco - II - idade à primeira relação sexual: análise exploratória. In: XIV Encontro Nacional de Estudos Populacionais. Campinas: Associação Brasileira de Estudos Populacionais; 2004. p. 1-17.

29. Figueiredo R, Andalaft Neto J. Uso de contracepção de emergência e camisinha entre adolescentes e jovens. Revista da SOGIA-BR 2005; 6:2.

30. Nunes MT. Conhecimento e utilização da contracepção de emergência em alunas do ensino secundário em Guimarães. Revista Portuguesa de Clínica Geral 2005; 21:247-56.

31. Aiken AM, Gold MA, Parker AM. Changes in young women's awareness, attitudes, and perceived barriers to using emergency contraception. J Pediat Adolesc Gynecol 2005; 18:25-32.

32. Prinstein MJ, Wang SS. False consensus and adolescent peer contagion: examining discrepancies between perceptions and actual reported levels of friends deviant and health risk behaviors. J Abnormal Child Psychol 2005; 33:293-306.

33. Wynn LL, Trussell J. The social life of emergency contraception in the United States: disciplining pharmaceutical use, disciplining sexuality, and zygotic bodies. Med Anthropol Q 2006; 20:297-320.

34. Branco VC. Os adolescentes nos serviços de saúde. Saber viver: adolescência e AIDS. Experiências e Reflexões sobre o Tema 2004; jan. http:/ / www.saber viver.org.br/index.php?g_edicao=servicossaude profissionais.

Recebido em 14/Fev/2008

Versão final reapresentada em 03/Jun/2008 Aprovado em 25/Ago/2008 